\section{Retard de croissance intra-utérin et dysplasie bronchopulmonaire}

Elodie Zana-Taïeb, Pierre-Henri Jarreau
Service de médecine et réanimation néonatales de Port-Royal, Hôpital Cochin, 53, avenue de l'Observatoire, 75014 Paris, France; Inserm U767, Fondation PremUP, Paris, France. pierre-henri.jarreau@cch.aphp.fr
$>$ Le retard de croissance intra-utérin (RCIU), défini comme une croissance inférieure au $10^{\mathrm{e}}$ percentile pour l'âge gestationnel, est une des principales pathologies faisant poser l'indication d'un accouchement prématuré. La cause la plus fréquente de RCIU est la pré-éclampsie maternelle (20 à $30 \%$ des cas), puis viennent les causes génétiques et infectieuses (10 à $30 \%$ ). Pendant la vie intra-utérine, les fœtus atteints de RCIU sont hypoxiques et en situation de malnutrition. À l'heure actuelle, il n'existe pas de stratégie préventive ou thérapeutique du RCIU. Les nouveau-nés atteints de RCIU (hypotrophes à la naissance) sont à risque de présenter de multiples pathologies à court et à long terme. En période néonatale, ces enfants, comparés aux enfants eutrophes de même terme, ont une mortalité augmentée et plus de difficultés d'adaptation à la vie post-natale. Sur le plan respiratoire, ils présentent une incidence plus importante de maladie des membranes hyalines ou d'hypertension artérielle pulmonaire primitive [15]. Ils ont aussi un risque plus élevé de complications multiples: thrombopénie, polyglobulie, intolérance glucidique, entérocolite ulcéro-nécrosante [1]. Plus tard, ces enfants présentent plus d'anomalies de la croissance et du développement psychomoteur [1]. À long terme, les antécédents d’hypotrophie par RCIU semblent «programmer» le développement d'un phénotype dit «économe » avec une augmentation de la prise alimentaire et une diminu- tion de la consommation d'énergie [1]. Ceci aboutit à l'âge adulte, surtout en cas d'apports caloriques non limités, à l'apparition d'une obésité et d'autres manifestations du syndrome métabolique [2].

Le RCIU a été mis en évidence plus récemment comme un facteur de risque indépendant de dysplasie bronchopulmonaire (DBP) (voir Encadré). Une étude australienne et néo-zélandaise retrouve, dans une population de terme identique constituée de plus de 11000 enfants nés entre 1998 et 2001, un risque augmenté de près d'un facteur 6 [3]. Une étude européenne récente en population (étude MOSAIC) dans 10 pays, regroupant plus de 4500 enfants nés en 2003, observe, à nouveau, un risque relatif de DBP augmenté de près d'un facteur 6 chez les hypotrophes [4]. Le rôle du poids de naissance est encore plus important chez les enfants nés avant 28 SA (semaines d'aménorrhée), dont le risque de DBP est augmenté, quand le poids de naissance est inférieur au poids médian [5]. Plusieurs études réalisées dans l'enfance et à l'âge adulte chez des patients porteurs d'un RCIU ont montré une anomalie persistante de la fonction respiratoire étudiée par des explorations fonctionnelles. Ainsi, chez des nourrissons de 10 mois (6-24 mois), on observe une augmentation de la résistance des voies aériennes chez les anciens hypotrophes [6].

La pré-éclampsie étant une pathologie exclusivement humaine, plusieurs modèles expérimentaux ont été déve- loppés pour étudier les conséquences pulmonaires du RCIU. Chez le mouton et le rat, des modèles d'insuffisance placentaire (embolisation ombilicoplacentaire) sont utilisés [7]. Chez le rat ou la souris, un RCIU peut également être induit par une restriction calorique et/ou protidique maternelle, un stress maternel (perturbation du rythme circadien) ou l'injection de L-NAME ( $\mathrm{N}$-nitro-L-arginine methyl ester, un inhibiteur de la synthèse de NO [nitric oxide] qui est un puissant vasodilatateur) [7]. Les résultats des différentes études sont discordants. G.S. Maritz a montré, chez l'agneau de 8 semaines ayant souffert d'un RCIU, une diminution du nombre d'alvéoles avec un élargissement de celles-ci, persistant chez I'animal adulte [8]. D'autres études chez l'agneau ont également montré des anomalies des voies aériennes (trachée et bronches) chez les animaux porteurs d'un RCIU [9]. Dans un modèle de RCIU induit par l'injection de L-NAME chez le rat, il a été montré une diminution de la surface alvéolaire à la naissance, persistant à 7 jours de vie post-natale mais totalement corrigée à 21 jours de vie (à la fin de l'alvéolisation) [10]. Nous avons comparé différents modèles de RCIU chez le raton. Nos résultats confirment les résultats obtenus par V. Diaz mais, en induisant un RCIU par une restriction protidique maternelle pendant toute la grossesse, nous observons une diminution de la surface alvéolaire dès 10 jours de vie, qui persiste ensuite. Associées aux anomalies de 


\section{LA DYSPLASIE BRONCHOPULMONAIRE}

Pierre-Henri Jarreau, Christophe Delacourt

La dysplasie bronchopulmonaire (DBP) est une pathologie décrite initialement à la fin des années 1960 comme la principale séquelle respiratoire des grands prématurés. Sur le plan respiratoire, l'immaturité initiale de ces enfants est caractérisée par un développement pulmonaire qui se situe à un stade très précoce et par un déficit en surfactant, substance tensio-active qui permet de maintenir les alvéoles ouverts en fin d'expiration. L'absence de surfactant conduit à la «maladie des membranes hyalines», principale pathologie respiratoire du prématuré, qui se traite par la ventilation mécanique et l'administration de surfactant directement dans les poumons. La DBP est le développement d'une pathologie respiratoire chronique et est définie par la nécessité d'un apport en oxygène ou d'un support respiratoire (ventilation mécanique) chez des enfants prématurés 28 jours après la naissance. L'évaluation de l'état respiratoire à 36 semaines d'âge post-menstruel ( $S A$, semaines d'aménorrhée), donc au-delà des 28 jours de vie si l'enfant est né avant 32 SA, caractérise sa gravité et est mieux corrélée au devenir respiratoire à moyen et long terme. Les enfants les plus sévèrement atteints sont en effet exposés à des réhospitalisations multiples pendant les 2 premières années de vie, le plus souvent pour une cause respiratoire. À long terme, leur fonction respiratoire peut être altérée et l'on observe plus de diagnostics d'«asthme». Enfin, la DBP a des conséquences sur le développement neurologique.

C'est avant tout une pathologie de la grande prématurité (terme inférieur ou égal à 32 SA, soit 7 mois de grossesse) qui représente $1,5 \%$ des naissances en France. La DBP survient chez environ $15 \%$ à $20 \%$ de ces enfants nés en Europe, ce taux semblant un peu plus élevé en Amérique du Nord. Elle a longtemps été considérée comme la conséquence de la prise en charge des détresses respiratoires néonatales. Les progrès réalisés dans le domaine n'ont pas apporté de diminution spectaculaire de sa fréquence mais ont permis une diminution des agressions subies parallèlement à l'augmentation de la survie de prématurés de plus en plus immatures, amenant une modification des lésions observées. Ceci est à l'origine du concept de «nouvelle»DBP, correspondant aux formes moins sévères, observées notamment depuis I'utilisation large du surfactant.

Il existe néanmoins de nombreux points communs entre les formes « ancienne » et «nouvelle », qui sont caractérisées par un arrêt de l'alvéolisation, aboutissant à un nombre d'alvéoles diminué par rapport à ce qu'il devrait être. En effet, la dernière phase du développement pulmonaire est le stade «alvéolaire», qui commence peu avant la naissance (vers 36 SA) et va se poursuivre au cours des deux premières années de la vie. Les grands prématurés naissent donc à un stade qui précède le développement alvéolaire, mais celui-ci est compromis par les évènements qui l'ont précédé. La DBP est donc avant tout une pathologie du développement pulmonaire distal, potentiellement aggravée par une réponse inadaptée aux agressions extérieures.

Les données épidémiologiques traduisent imparfaitement la réalité : la DBP a certainement diminué en fréquence et en gravité chez les « moins » immatures, mais reste peu sensible aux progrès de la prise en charge chez les « extrêmes prématurés » nés avant 28 SA. Plusieurs facteurs sont en cause : l'importance de l'immaturité pulmonaire, en particulier chez les enfants trop petits pour leur terme (hypotrophes), le rôle des phénomènes inflammatoires locaux qui sont la voie finale commune de la majeure partie des agressions, et l'importance de facteurs de risque génétiques qui pourraient expliquer cette persistance d'un taux incompressible de DBP malgré l'amélioration constante des soins

Ces 3 facteurs ont été ou sont l'objet de thèses réalisées dans le cadre de la fondation de coopération scientifique PremUP « Grossesse et Prématurité » et leurs auteurs en font le point dans ce dossier [1-3].

1. Zana-Taïeb $\varepsilon$, Jarreau PH. Retard de croissance intra-utérin et dysplasie bronchopulmonaire. Med Sci (Paris) $2013 ; 29: 826-8$.

2. Hadchouel A, Delacourt C. Dysplasie bronchopulmonaire et génétique. Med Sci (Paris) $2013 ; 29: 821-3$.

3. Lopez $\varepsilon$, Jarreau PH. Inflammation et dysplasie bronchopulmonaire. Med Sci (Paris) $2013 ; 29: 823-5$.
I'alvéolisation, l'existence d'un dysfonctionnement des cellules endothéliales des artères pulmonaires a été démontré très récemment avec une diminution de la densité vasculaire de $44 \%$ par rapport aux témoins [11].

Les mécanismes physiopathologiques de ces anomalies restent mal compris et les résultats sont là encore discordants selon le modèle de RCIU étudié. Des facteurs de croissance ou des facteurs de transcription comme PPAR $\gamma$ [12] semblent participer aux troubles de l'alvéolisation. Ainsi, L.A. Joss-Moore observe une diminution des transcrits des gènes régulant la voie de l'élastine chez le rat [13] alors que M.L. Cock, chez le mouton, ne retrouve pas de différence [14].

En conclusion, le RCIU est un facteur de risque indépendant de développer une DBP et ces anomalies de la fonction respiratoire persistent chez l'adulte. Les résultats des modèles expérimentaux sont discordants et les mécanismes physiopathologiques restent à élucider. $\diamond$ In utero growth retardation and bronchopulmonary dysplasia

\section{LIENS D'INTÉRÊT}

Les auteurs déclarent n'avoir aucun lien d'intérêt concernant les données publiées dans cet article.

\section{RÉFÉRENCES}

1. Rosenberg A. The IUGR newborn. Semin Perinatol $2008 ; 32: 219-24$.

2. Barker DJ, Eriksson JG, Forsen T, Osmond C. Fetal origins of adult disease: strength of effects and biological basis. Int J Epidemiol 2002 ; 31 : 1235-9.

3. Henderson-Smart DJ, Hutchinson JL, Donoghue DA, et al. Prenatal predictors of chronic lung disease in very preterm infants. Arch Dis Child Fetal Neonatal Ed $2006 ; 91:$ F40-5

4. Zeitlin J, દl Ayoubi M, Jarreau PH, et al. Impact of fetal growth restriction on mortality and morbidity in a very preterm birth cohort. J Pediatr ; 157 : 733-9 el.

5. Kewitz G, Wudel S, Hopp H, et al. Below median birth weight in appropriate-for-gestational-age preterm infants as a risk factor for bronchopulmonary dysplasia. J Perinat Med 2008 ; 36 : 359-64.

6. Greenough A, Yuksel B, Cheeseman P. Effect of in utero growth retardation on lung function at followup of prematurely born infants. Eur Respir J 2004 ; $24: 731-3$. 


\section{RÉFÉRENCES}

7. McMillen IC, Adams MB, Ross JT, et al. Fetal growth restriction: adaptations and consequences. Reproduction 2001 ; 122 : 195-204.

8. Maritz GS, Cock ML, Louey S, Suzuki K, Harding R. Fetal growth restriction has long-term effects on postnatal lung structure in sheep. Pediatr Res 2004 ; 55 : 287-95

9. Wignarajah D, Cock ML, Pinkerton KE, Harding R. Influence of intrauterine growth restriction on airway development in fetal and postnatal sheep. Pediatr Res $2002 ; 51: 681-8$.
10. Diaz V, Lebras-Isabet MN, Denjean A. Effect of Nomega-nitro-L-arginine methyl ester-induced intrauterine growth restriction on postnatal lung growth in rats. Pediatr Res $2005 ; 58: 557-61$.

11. Rozance PJ, Seedorf GJ, Brown A, et al. Intrauterine growth restriction decreases pulmonary alveolar and vessel growth and causes pulmonary artery endothelial cell dysfunction in vitro in fetal sheep. Am J Physiol Lung Cell Mol Physiol 2011 ; 301 : L860-71.

12. Joss-Moore LA, Wang $Y$, Baack ML, et al. IUGR decreases PPARgamma and SETD8 Expression in neonatal rat lung and these effects are ameliorated by maternal DHA supplementation. Early Hum Dev $2010 ; 86: 785-91$.

13. Joss-Moore LA, Wang $Y, y_{u} X$, et al. IUGR decreases elastin mRNA expression in the developing rat lung and alters elastin content and lung compliance in the mature rat lung. Physiol Genomics $2011 ; 43$ : 499-505

14. Cock ML, Joyce BJ, Hooper SB, et al. Pulmonary elastin synthesis and deposition in developing and mature sheep: effects of intrauterine growth restriction. Exp Lung Res $2004 ; 30$ : 405-18.

15. Perros F, Humbert M, Cohen-Kaminsky S. Hypertension artérielle pulmonaire : un parfum d'auto-immunité. Med Sci (Paris) $2013 ; 29: 607-16$.

\section{NOUVELLE}

\section{À propos du développement cérébral des prématurés}

François Rousseau ${ }^{1}$, Nadine Girard ${ }^{2,3}$

\author{
${ }^{1}$ ICube, UMR 7357, université de Strasbourg, \\ CNRS, 300, boulevard Sébastien Brant, 67412 IIlkirch, France; \\ ${ }^{2}$ Centre de résonance magnétique biologique et médicale \\ (CRMBM), UMR 7339, université Aix-Marseille, \\ CNRS, 13005 Marseille, France ; \\ ${ }^{3}$ Hôpital la Timone, université de la Méditerranée, 13005 \\ Marseille, France.
}

rousseau@unistra.fr
> Chaque année, environ $10 \%$ des bébés dans le monde naissent prématurément (soit avant 37 semaines d'aménorrhée). La prématurité est la principale cause de décès chez les nouveau-nés et un nombre conséquent de ceux qui survivent souffrent d'incapacité à vie, notamment concernant l'apprentissage, la vision ou l'audition (rapport Born too soon de l'Organisation mondiale de la santé [OMS] paru en $2012^{1}$ ). Les progrès réalisés dans la prise en charge de ces enfants ont contribué à améliorer la survie des grands prématurés. Cependant, l'étude EPIPAGE (enquête épidémiologique sur les petits âges gestationnels), lancée en France en 1997, a montré que $42 \%$ des enfants nés entre 24 et 28 semaines d'âge gestationnel et $31 \%$ de ceux qui sont nés entre 29 et 32 semaines ont besoin d'une prise en charge médicale spécialisée spécifique pendant les 5 premières années, contre $16 \%$ de ceux qui sont nés à terme [1]. Il est difficile de prévoir les risques neurologiques que courent les prématurés survivants

${ }^{1}$ Le rapport peut être téléchargé à l'adresse suivante : http://www.who.int/pmnch/media/news/2012/preterm_ birth_report/en/ à long terme, ce qui souligne l'importance d'approfondir nos connaissances sur l'impact d'une naissance prématurée sur le développement cérébral.

\section{Des moyens limités d'étude}

\section{du développement cérébral}

Le développement du cerveau suit une succession d'étapes régies par une interaction complexe entre des milliers de gènes et des facteurs environnementaux. Connaître le déroulement temporel exact de ces événements en situation normale permettrait de mieux appréhender les mécanismes à l'origine des séquelles provoquées par des altérations de ce développement lors de la prématurité, et donc de mieux adapter les techniques de prise en charge des prématurés. De multiples processus neurobiologiques interviennent dans la plage temporelle correspondant à une naissance prématurée (de 24 à 37 semaines d'aménorrhée) : migration neuronale, bourgeonnement dendritique, formation des synapses, prolifération des cellules gliales, apoptose neuronale, etc. [2].

Étudier un phénomène aussi complexe que la maturation cérébrale est délicat. Les investigations post-mortem per- mettent d'obtenir des informations à des échelles très fines, mais elles ont une portée réduite car elles sont limitées à une anatomie (souvent pathologique) analysée à un instant précis du développement. Les informations concernant l'aspect fonctionnel ainsi que la dynamique du développement sont perdues. Les études chez l'animal permettent de s'affranchir de ces limites mais l'extrapolation des résultats observés chez un animal au cerveau humain peut être délicate. Les techniques d'imagerie non invasives, telles que l'échographie, l'imagerie par résonance magnétique (IRM), l'électro-encéphalographie (દદG) et la magnéto-encéphalographie (MEG) permettent, de façon complémentaire, d'étudier in vivo le cerveau à ce stade du développement au niveau anatomique et fonctionnel, ainsi que les dynamiques temporelles associées.

Dans ce contexte, I'IRM de diffusion est une technique d'imagerie prometteuse car elle permet d'estimer en chaque point du cerveau la distribution des directions de diffusion des molécules d'eau. En supposant que cette diffusion soit principalement contrainte par les fibres nerveuses, il est alors possible 